\title{
Theanine, an antitumor promoter, induces apoptosis of tumor cells via the mitochondrial pathway
}

\author{
YINQIANG XIN ${ }^{1 *}$, PEILING BEN ${ }^{1,2 *}$, QI WANG ${ }^{1}$, YANYAN ZHU ${ }^{1}$, ZHIMIN YIN $^{1}$ and LAN LUO ${ }^{3}$ \\ ${ }^{1}$ Jiangsu Province Key Laboratory for Molecular and Medical Biotechnology, College of Life Sciences, \\ Nanjing Normal University, Nanjing, Jiangsu $210023 ;{ }^{2}$ Department of Biochemistry, \\ Chuzhou City Vocation College, Chuzhou, Anhui 239000; ${ }^{3}$ State Key Laboratory of Pharmaceutical Biotechnology, \\ School of Life Sciences, Nanjing University, Nanjing, Jiangsu 210093, P.R. China
}

Received May 5, 2016; Accepted March 28, 2017

DOI: $10.3892 / \mathrm{mmr} .2018 .9459$

\begin{abstract}
Theanine, an active component of green tea (Camellia sinensis), is considered a modulator of chemotherapy. To further investigate the anticancer activity of theanine, the present study investigated the cytotoxic effect of theanine at the concentration of $600 \mu \mathrm{g} / \mathrm{ml}$, in the human HepG2 hepatoblastoma and HeLa adenocarcinoma cell lines, in comparison with the normal L02, H9c2 and HEK293 cell lines using a MTT assay. It was found that theanine induced cell death in the tumor cells, but not in the normal cells. Notably, when glutamine was restricted or reduced in the cell culture medium, the cell death induced by theanine was significantly enhanced. A terminal deoxynucleotidyl-transferase-mediated dUTP nick end labeling assay indicated that DNA damage was induced in theanine-treated HepG2 cells. Further experiments demonstrated that theanine caused HepG2 cell apoptosis through the mitochondrial pathway, with a loss of membrane potential and the release of apoptosis-inducing factor, endonuclease $\mathrm{G}$ and cytochrome c. Western blot analysis and caspase activity detection also revealed that caspase- 9 and caspase- 3 were activated, whereas caspase- 8 remained inactive. These observations suggested that theanine exerted potent cytotoxicity on tumor cells when glutamine was restricted.
\end{abstract}

Correspondence to: Professor Zhimin Yin, Jiangsu Province Key Laboratory for Molecular and Medical Biotechnology, College of Life Sciences, Nanjing Normal University, 1 Wenyuan Road, Nanjing, Jiangsu 210023, P.R. China

E-mail: yinzhimin@njnu.edu.cn

Professor Lan Luo, State Key Laboratory of Pharmaceutical Biotechnology, School of Life Sciences, Nanjing University, 163 Xianlin Avenue, Nanjing, Jiangsu 210093, P.R. China

E-mail: lanluo@nju.edu.cn

*Contributed equally

Key words: theanine, glutamine, apoptosis, caspase-9, mitochondrial pathway

\section{Introduction}

L-theanine $\left(\gamma\right.$-glutamylethylamide; $\left.\mathrm{C}_{7} \mathrm{H}_{14} \mathrm{~N}_{2} \mathrm{O}_{3}\right)$ is a water-soluble, edible, non-proteinogenic amino acid found principally in Camellia sinensis, the source of green tea (1). Previous studies in rats have indicated that orally administered theanine is absorbed through the intestinal tract, and hydrolyzed to glutamic acid and ethylamine in the rat kidney $(2,3)$. Theanine has been comprehensively examined for its effects on mood-modulation and neuroprotection, and for its lipid-lowering, antihypertensive, chemosensitizing and hepatoprotective properties (4-8).

The pathogenesis of several diseases, including cancer, is closely connected with abnormal apoptotic cell death (9). The regulation of apoptosis and associated mediators, which trigger or inhibit cell death, have become the foundation for establishing therapeutic strategies in cancer. Numerous studies have demonstrated that active components from green tea, including catechins, theaflavins and polyphenols, can induce apoptosis in several tumor cell lines (10-12). Theanine, considered a novel biochemical modulator for chemotherapeutics, can also suppress tumor cell invasion (13). It has been reported that theanine suppressed the growth of A549 human non-small cell lung cancer and K562 leukemic cell lines in a dose- and time-dependent manner (14). Sugiyama and Sadzuka (15) reported that theanine significantly enhanced the inhibitory effect of doxorubicin (DOX) on tumor growth in M5076 ovarian sarcoma-bearing mice, and increased the concentration of DOX in tumor cells. Further investigation has demonstrated that theanine increases the antitumor efficacy of DOX via the inhibition of glutamate transporters (16).

The aim of the present study was to investigate the molecular mechanisms underlying the antitumor actions of theanine. In the present study, theanine exhibited an enhanced apoptotic efficacy on HepG2 cells when glutamine, an analogue of theanine, was restricted in the cell culture medium. This suggested that glutamine interfered with the action of theanine in the tumor cells. It was also demonstrated that theanine exerted an apoptotic effect on the HepG2 cells via mitochondrial pathways and the activation of caspase-9. These findings indicated theanine is a valuable antitumor component in food and in clinical treatment. 


\section{Materials and methods}

Antibodies and reagents. Polyclonal rabbit antibodies against poly (ADP-ribose) polymerase (PARP; cat no. 5625), caspase-3 (cat no. 9662), caspase-8 (cat no. 4790), caspase-9 (cat no. 9508), cytochrome c (cat no. 4272) and $\beta$-actin (cat no. 4970) were obtained from Cell Signaling Technology, Inc. (Beverly, MA, USA). Monoclonal mouse antibody against cyclooxygenase (COX) IV (cat no. ab16056), polyclonal rabbit antibodies against apoptosis-inducing factor (AIF; cat no. ab32516) and endonuclease G (EndoG; cat no. ab9647) were from Abcam (Cambridge, UK). L-theanine, L-glutamine and 3-(4,5-dimethylthiazol-2-yl)-2,5-diphenyl tetrazolium bromide (MTT) were purchased from Sigma-Aldrich; Merck KGaA (Darmstadt, Germany). 4,6-diamidino-2-phenylindole was from Invitrogen; Thermo Fisher Scientific, Inc. (Waltham, MA, USA). Z-LEHD-FMK, Z-IETD-FMK and Z-VAD-FMK were provided by Merck KGaA.

Cells and culture conditions. Human hepatoblastoma HepG2 cells, human cervical carcinoma HeLa cells, human hepatic L02 cells, human embryonic kidney 293 (HEK293) cells and rat cardiac myoblast $\mathrm{H} 9 \mathrm{c} 2$ cells were purchased from the Cell Bank of Type Culture Collection of Chinese Academy of Sciences (Shanghai, China). Cells were cultured in Dulbecco's modified Eagle's medium (DMEM; Hyclone; GE Healthcare Life Sciences, Logan, UT, USA) supplemented with $10 \%$ fetal bovine serum (FBS; Hyclone; GE Healthcare Life Sciences), $100 \mathrm{U} / \mathrm{ml}$ penicillin and $100 \mu \mathrm{g} / \mathrm{ml}$ streptomycin at $37^{\circ} \mathrm{C}$ with $5 \% \mathrm{CO}_{2}$. Glutamine restriction or deprivation were performed by culturing cells with glutamine-containing $(2.0 \mathrm{mM})$ or glutamine-free DMEM (Hyclone; GE Healthcare Life Sciences) supplemented with 10\% FBS for $24 \mathrm{~h}$.

Cell viability/toxicity assay. HepG2, HeLa, A549, L02 and HEK293 cells were seeded into 96-well plates at a density of $5 \times 10^{3}$ cells/well $24 \mathrm{~h}$ prior to treatment. Following treatment with various concentrations of L-theanine $(0-600 \mu \mathrm{g} / \mathrm{ml})$ at $37^{\circ} \mathrm{C}$ for $24 \mathrm{~h}$, cell viability was determined using an MTT assay. Briefly, $15 \mu \mathrm{l}(5 \mathrm{mg} / \mathrm{ml})$ MTT working solution were added into each well and, following incubation at $37^{\circ} \mathrm{C}$ for $4 \mathrm{~h}$, the MTT solution was removed and $200 \mu \mathrm{l}$ DMSO were added to dissolve the crystals. The absorbance of each well at $570 \mathrm{~nm}$ was measured using a Synergy2 Multi-Mode microplate reader (BioTek Instruments, Inc., Winooski, VT, USA). The precise determination of cytotoxicity was achieved using a CytoTox-Glo ${ }^{\mathrm{TM}}$ cytotoxicity assay (Promega Corporation, Madison, WI, USA) to measure cell viability, according to the manufacturer's protocol.

Terminal deoxynucleotidyl transferase dUTP nick end labeling (TUNEL) assay. HepG2 cells were seeded at a density of $5 \times 10^{5}$ cells/well $24 \mathrm{~h}$ prior to treatment. Following treatment with various concentrations of $\mathrm{L}$-theanine $(0-600 \mu \mathrm{g} / \mathrm{ml})$ at $37^{\circ} \mathrm{C}$ for $24 \mathrm{~h}$, a TUNEL assay was performed using the Guava TUNEL kit according to the manufacturer's protocol. The cells were added to the Guava EasyCyte $^{\mathrm{TM}}$ system, and the data were analyzed using Guava TUNEL software version 2.0 (Guava Technologies, Inc., Hayward, CA, USA).
MultiCaspase apoptosis detection. HepG2 cells were seeded at a density of $5 \times 10^{5}$ cells/well $24 \mathrm{~h}$ prior to treatment. Following treatment with various concentrations of L-theanine $(0-600 \mu \mathrm{g} / \mathrm{ml})$ at $37^{\circ} \mathrm{C}$ for $24 \mathrm{~h}$, the number of cells in the various stages of the apoptotic pathway were determined via MultiCaspase apoptosis detection (Guava Technologies, Inc.). The assay was performed according to the manufacturer's protocol and the stained cells were analyzed using the Guava EasyCyte ${ }^{\mathrm{TM}}$ flow cytometer. A fluorochrome-conjugated inhibitor of caspases, termed sulforhodamine-valyl-alanyl-aspartyl-fluoromethylketone, and the cell impermeant DNA dye 7-aminoactinomycin (7-AAD) were used to distinguish between non-apoptotic living cells, early/mid apoptotic stage cells, late apoptotic/dying cells and dead cells. Data were analyzed using the Guava TUNEL Software version 2.0 (Guava Technologies, Inc.).

Western blot analysis. HepG2 cells were seeded at a density of $5 \times 10^{5}$ cells/well $24 \mathrm{~h}$ prior to treatment. Following treatment with various concentrations of L-theanine $(0-600 \mu \mathrm{g} / \mathrm{ml})$ at $37^{\circ} \mathrm{C}$ for $12 \mathrm{~h}$, cells were lysed for $30 \mathrm{~min}$ on ice in a lysis buffer containing $135 \mathrm{mM} \mathrm{NaCl}, 20 \mathrm{mM}$ Tris (pH 7.5), $25 \mathrm{mM}$ $\beta$-glycerophoshate, 2 mM EDTA, 2 mM DTT, 2 mM sodium pyrophosphate, $10 \%$ glycerol, $1 \%$ Triton X-100, $10 \mathrm{mM} \mathrm{NaF}$, $1 \mathrm{mM}$ sodium orthovanadate and $1 \mathrm{mM}$ PMSF, supplemented with complete protease inhibitor cocktail (Roche Applied Science, Indianapolis, IN, USA). The lysates were centrifuged $(15,000 \mathrm{x} \mathrm{g})$ at $4^{\circ} \mathrm{C}$ for $15 \mathrm{~min}$. Equal amounts of extracted protein samples $(20 \mu \mathrm{g})$ were denatured in SDS, separated by $12 \%$ SDS-PAGE, and transferred onto nitrocellulose membranes (GE Healthcare Life Sciences). Membranes were blocked with $5 \%$ non-fat dry milk in Tris-buffered saline with $0.1 \%$ Tween-20 for $2 \mathrm{~h}$ at room temperature. Subsequently, membranes were incubated with the following primary antibodies overnight at $4^{\circ} \mathrm{C}$ : Anti-caspase-3 $(1: 1,000)$, anti-PARP $(1: 1,000)$, anti-caspase-9 (1:1,000), anti-caspase-8 $(1: 1,000)$, anti-COX IV $(1: 1,000)$, anti-cytochrome c $(1: 1,000)$, anti-AIF $(1: 1,000)$, anti-EndoG $(1: 1,000)$ and anti- $\beta$-actin $(1: 1,000)$. The protein bands were visualized using the LI-COR Odyssey infrared imaging system, according to the manufacturer's protocol, using an IRDye800 fluorophore-conjugated goat anti-rabbit IgG (cat no. 926-32211; 1:5,000; LI-COR Biosciences, Lincoln, NE, USA) or anti-mouse IgG (cat no. 926-32210; 1:5,000; LI-COR Biosciences) secondary antibody in the dark at room temperature for $1 \mathrm{~h}$. Blots were semi-quantified by densitometric analysis using the LI-COR Odyssey analytical software version 2.1 (LI-COR Biosciences).

Caspase activity measurement. HepG2 cells were seeded at a density of $5 \times 10^{5}$ cells/well $24 \mathrm{~h}$ prior to treatment. Following treatment with various concentrations of L-theanine $(0-600 \mu \mathrm{g} / \mathrm{ml})$ at $37^{\circ} \mathrm{C}$ for $12 \mathrm{~h}$, caspase- 8 and caspase- 9 activity was measured using the Caspase-Glo ${ }^{\circledR} 8$ assay and Caspase-Glo ${ }^{\circledR} 9$ assay, respectively (Promega Corporation). The assays were performed according to the manufacturer's protocol. The luminescence was measured on a Synergy2 Multi-Mode microplate reader (BioTek Instruments, Inc.).

Measurement of mitochondrial membrane potential (MMP). HepG2 cells were seeded at a density of $5 \times 10^{5}$ cells/well $24 \mathrm{~h}$ 


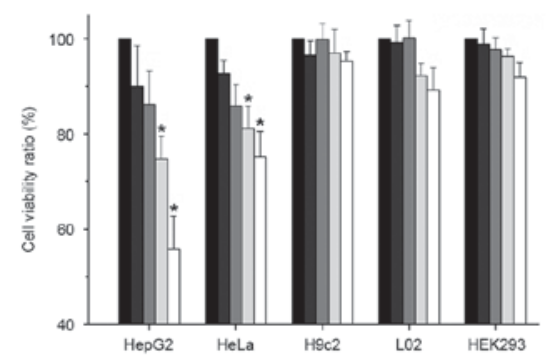

B

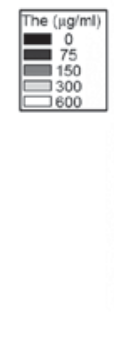

HepG2

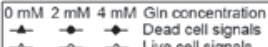

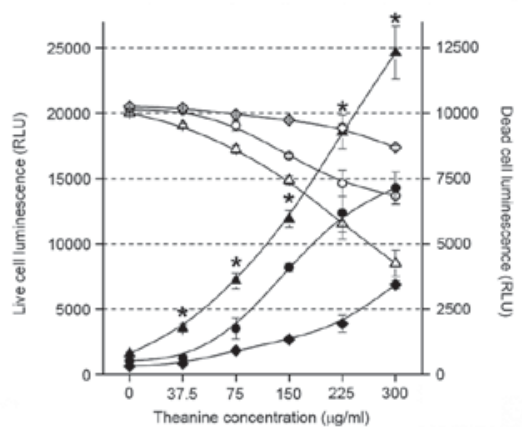
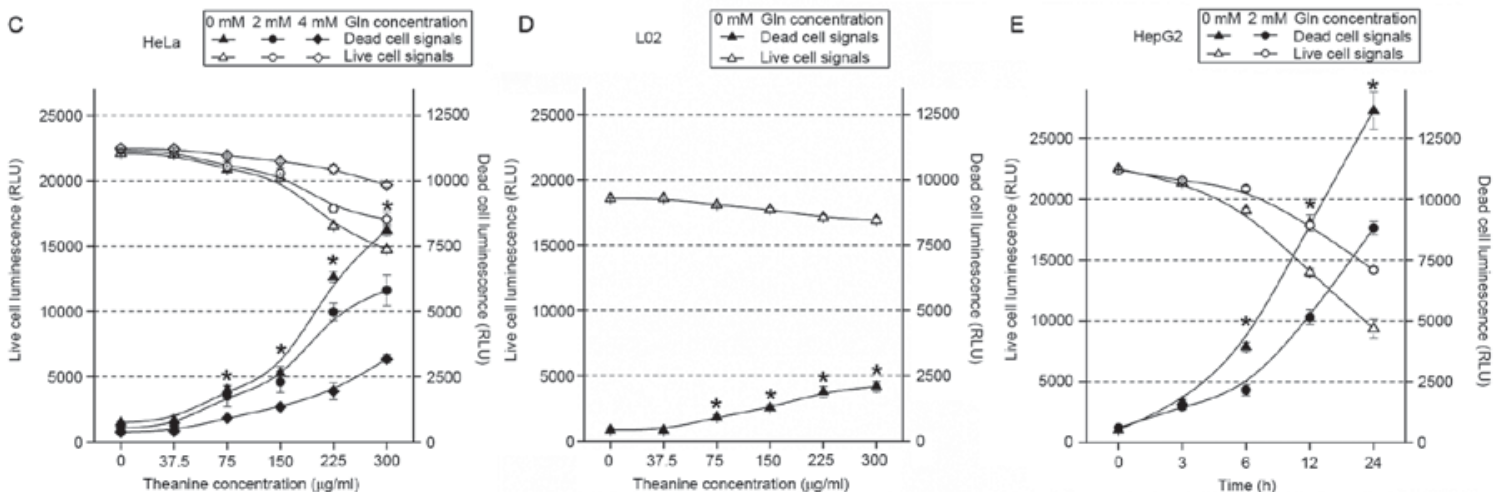

Figure 1. Effects of glutamine concentration on theanine-induced cytotoxicity. (A) HepG2, HeLa, H9c2, L02 and HEK293 cells were incubated with various concentrations of theanine, respectively. Cell viability was measured using a 3-(4,5-dimethylthiazol-2-yl)-2,5-diphenyl tetrazolium bromide assay. Data shown are representative of three independent experiments. ${ }^{*} \mathrm{P}<0.05$, compared with the untreated control. (B-E) HepG2, HeLa or L02 cells in glutamine-free or glutamine-restricted ( 2 or $4 \mathrm{mM}$ ) medium were treated with various concentrations of theanine for $24 \mathrm{~h}$ respectively. HepG 2 cells in glutamine-free or glutamine-restricted $(2 \mathrm{mM})$ media were treated with $300 \mu \mathrm{g} / \mathrm{ml}$ theanine for the indicated time. In all cases, theanine cytotoxicity was determined by a luminescence-based method as described. Each value is a mean \pm SD of three replicates. Gln, glutamine; The, theanine.

prior to treatment. Following treatment with various concentrations of L-theanine $(0-600 \mu \mathrm{g} / \mathrm{ml})$ at $37^{\circ} \mathrm{C}$ for $12 \mathrm{~h}$, the MMP of the HepG2 cells was assessed using the Guava EasyCyte ${ }^{\mathrm{TM}}$ MitoPotential $^{\mathrm{TM}}$ kit. In this assay, JC-1 cationic dye is used to evaluate alterations in MMP. The cell impermeant dye, 7-AAD, was used to simultaneously monitor cell membrane permeability alterations in apoptosis. The stained cells were analyzed using the Guava EasyCyte ${ }^{\mathrm{TM}}$ flow cytometer (Guava Technologies, Inc.).

Mitochondrial isolation. HepG2 cells were seeded at a density of $2 \times 10^{7}$ cells/well $24 \mathrm{~h}$ prior to treatment. Following treatment with various concentrations of L-theanine $(0-600 \mu \mathrm{g} / \mathrm{ml})$ at $37^{\circ} \mathrm{C}$ for $12 \mathrm{~h}$, intact mitochondria were separated from cytosolic components of the HepG2 cells for further protein analysis using a mitochondria isolation kit (cat no. 89874; Thermo Fisher Scientific, Inc.). Dounce homogenization and differential centrifugation were performed according to the manufacturer's protocol.

Statistical analysis. All experimental data are expressed as the mean \pm standard deviation of three independent experiments in each group. The statistical significance of the differences between the experimental groups was assessed by Student's t-test for pair-wise comparisons and one-way analysis of variance followed by Tukey's post-hoc test for multiple comparisons. Statistical analysis was performed using the SPPS software version 17.0 (SPSS, Inc., Chicago, IL, USA).
$\mathrm{P}<0.05$ was considered to indicate a statistically significant difference.

\section{Results}

Theanine-induced cancer cell death is potentiated by acute glutamine deprivation. To determine an anticancer activity of theanine, the present study detected the effect of theanine on tumor and normal cell viability. As shown in Fig. 1A, theanine significantly decreased the viability of the HepG2 and HeLa cells in a dose-dependent manner between from 75 and $600 \mu \mathrm{g} / \mathrm{ml}$. Theanine treatment at the concentration of $600 \mu \mathrm{g} / \mathrm{ml}$, resulted in decreases in viability of $\sim 5$ and $25 \%$ in the HepG2 and HeLa cells, respectively, compared with the untreated control cells. By contrast, reductions in viability of $<10 \%$ were observed in the H9c2, L02 and HEK293 cells. These data, together with previous findings (17) indicated that the anticancer activity of theanine was cell-type specific, and suggested that theanine possessed anticancer activity with almost no harmful effect on normal cells.

As theanine and glutamine are analogues, and are mutually exchangeable due to their similar structure, the present subsequently investigated whether glutamine present in the cell culture medium interfered with the cytotoxic effect of theanine on cancer cells. As it was noted that complete DMEM commonly contains $4 \mathrm{mM}$ glutamine, the present study used $2 \mathrm{mM}$ glutamine-containing DMEM or glutamine-free DMEM for incubation of the HepG2, HeLa and L02 cells in order to 

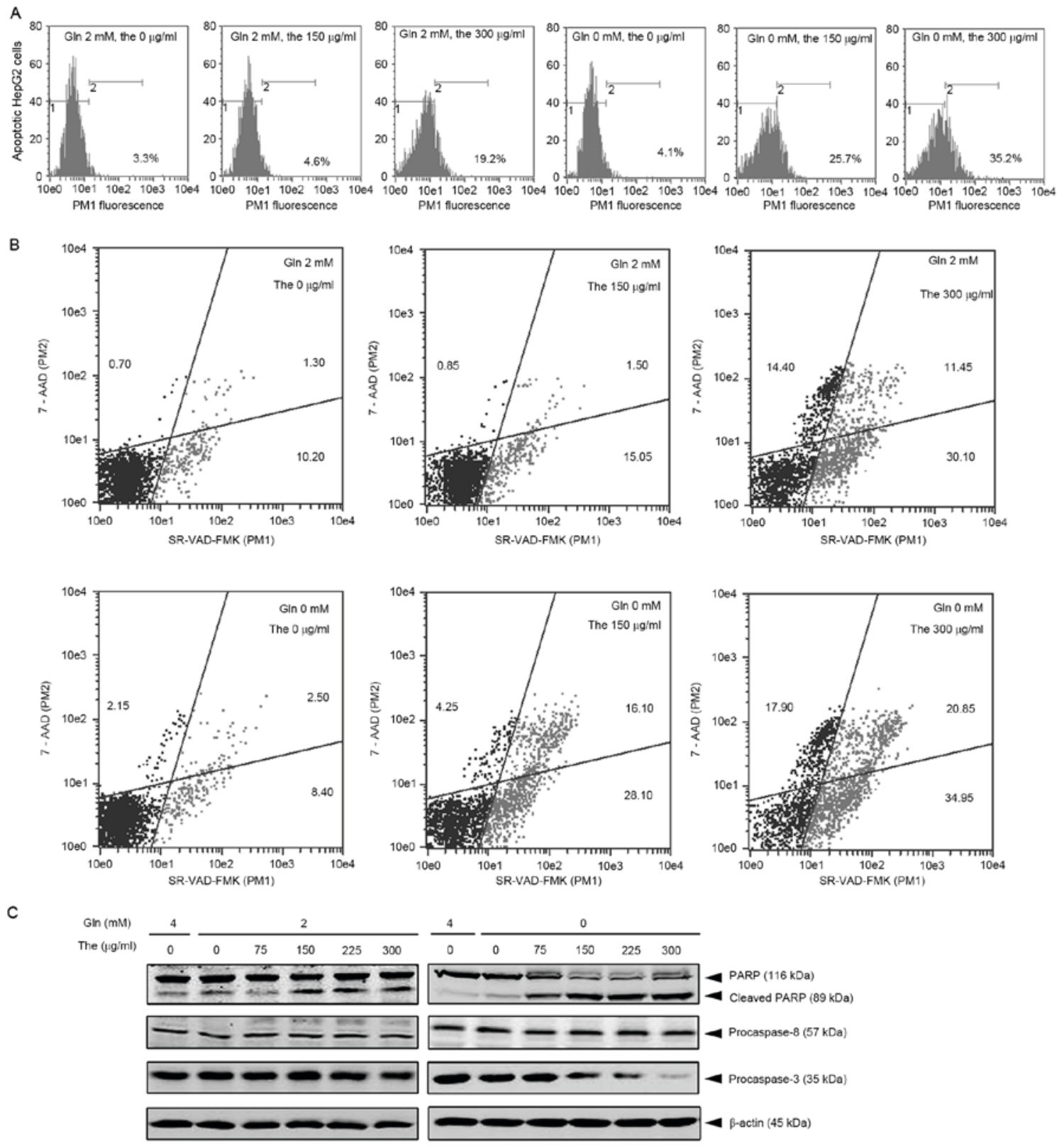

Figure 2. Apoptosis of HepG2 cells is induced by theanine. HepG2 cells in glutamine-free or glutamine-restriction ( $2 \mathrm{mM})$ media were treated with 0 , 150 or $300 \mu \mathrm{g} / \mathrm{ml}$ theanine for $24 \mathrm{~h}$. Percentages of apoptotic cells were assessed using (A) TUNEL analysis and (B) multicaspase detection, respectively. For TUNEL analysis, the average apoptotic ratio of each group is shown. For multicaspase detection, four quadrants denote non-apoptotic live cells (bottom-left), early/mid apoptotic stage cells (bottom-right), late apoptotic/dying cells (upper-right), and dead cells (upper-left). Data shown are representative of three independent experiments. (C) HepG2 cells in glutamine-free or glutamine-containing ( 2 or $4 \mathrm{mM}$ ) medium were treated with $300 \mu \mathrm{g} / \mathrm{ml}$ theanine for $24 \mathrm{~h}$, and cell lysates were prepared. The cleavage of PARP, caspase- 8 and caspase- 3 were determined using western blot analysis. Expression of $\beta$-actin was determined to confirm equal protein loading. Data shown are representative of three independent experiments. Gln, glutamine; The, theanine; TUNEL, terminal deoxynucleotidyl transferase dUTP nick end labeling; PARP, poly (ADP-ribose) polymerase.

achieve acute glutamine restriction or deprivation. The cells incubated under the acute glutamine restriction or deprivation conditions were treated with $0-300 \mu \mathrm{g} / \mathrm{ml}$ theanine for $24 \mathrm{~h}$. As shown in Fig. 1B, theanine led to apparent dose-dependent cell death of HepG2 cells, dependent on glutamine concentration in the culture medium. In addition to the decreased glutamine concentration, theanine-induced cell death increased. A similar effect of glutamine on theanine-induced cytotoxicity was also detected in the HeLa cells (Fig. 1C). However, neither glutamine restriction nor deprivation altered the effect of theanine on the L02 cells (Fig. 1D). Furthermore, under acute glutamine deprivation, theanine induced HepG2 cell death in a time-dependent manner (Fig. 1E). These data indicated that glutamine prevented theanine-induced cancer cell death.

Theanine induces HepG2 cell apoptosis under acute glutamine deprivation. To determine whether theanine-induced cytotoxicity was due to apoptosis, a series of classical apoptosis detection experiments were performed. The results of the TUNEL assay, using flow cytometric analysis, indicated DNA damage in the theanine-treated HepG2 cells (Fig. 2A). As the caspase family is pivotal in apoptosis (18), the present study 
A

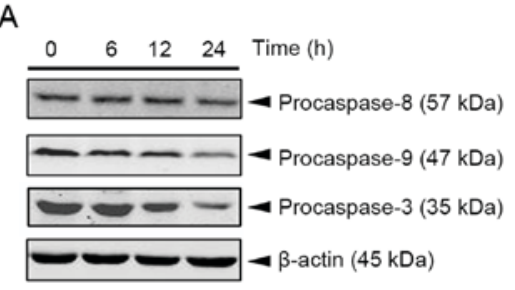

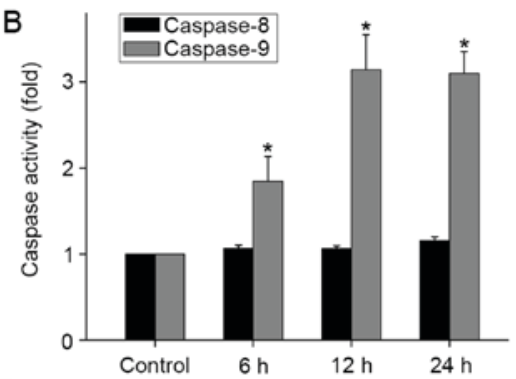

C

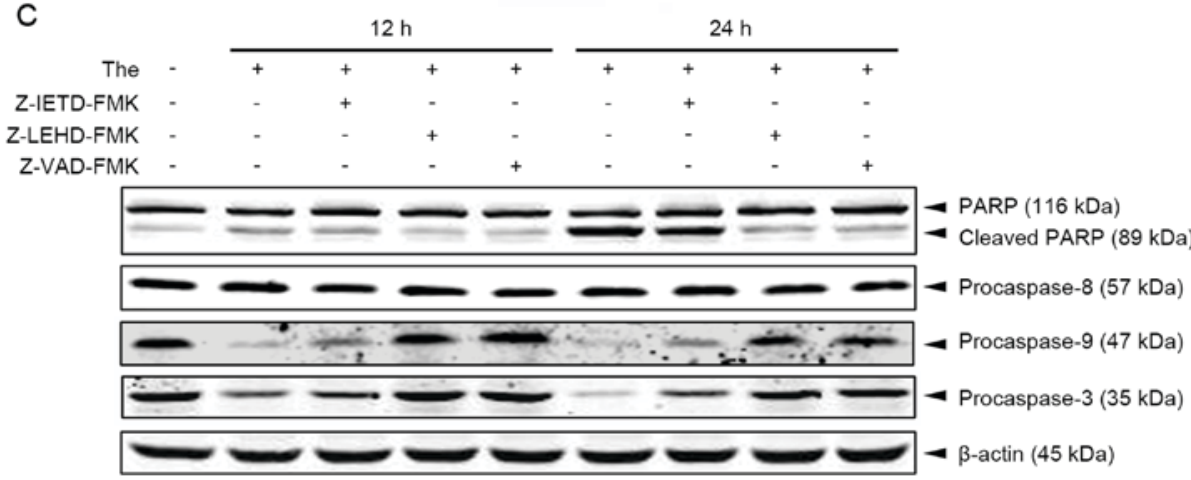

$D$

$12 \mathrm{~h}$

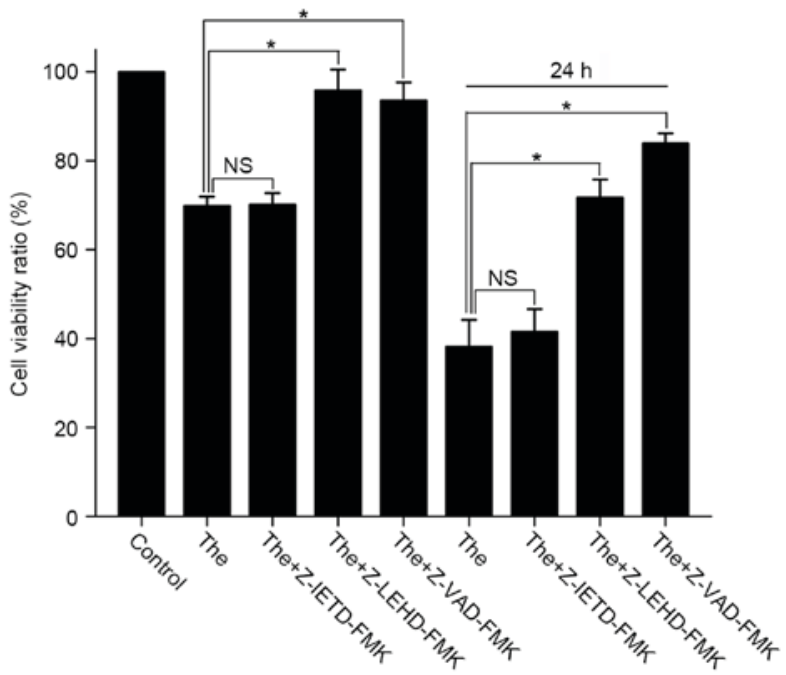

Figure 3. Caspase pathway activation in theanine-induced apoptosis. (A) HepG2 cells in glutamine-free media were treated with $300 \mu \mathrm{g} / \mathrm{ml}$ theanine for the indicated durations, respectively. Cell lysates were prepared, and the levels of caspase- 8 , caspase- 9 and caspase-3 were assessed using western blot analysis. Expression of $\beta$-actin was determined to confirm equal protein loading. Data shown are representative of three independent experiments. (B) Following treatment with $300 \mu \mathrm{g} / \mathrm{ml}$ theanine for the indicated time, activities of caspase- 8 and caspase-9 in the HepG2 cells were detected using a luminescence-based method. Data shown are representative of three independent experiments. ${ }^{*} \mathrm{P}<0.05$, compared with the untreated control. (C) HepG2 cells were exposed to $300 \mu \mathrm{g} / \mathrm{ml}$ theanine for 12 or $24 \mathrm{~h}$, accompanied with caspase-8 inhibitor Z-IETD-FMK, caspase-9 inhibitor Z-LEHD-FMK or pan-caspase inhibitor Z-VAD-FMK treatment, respectively, as indicated. Levels of caspase-8, caspase-9 and caspase-3 were determined using western blot analysis. Expression of $\beta$-actin was assessed to confirm equal protein loading. Data shown are representative of two independent experiments. (D) Cell viability was measured using a 3-(4,5-dimethylthiazol-2-yl)-2,5-diphenyl tetrazolium bromide assay. Cell viability without treatment was taken as 100\%. Data are presented as the mean \pm standard deviation of three independent experiments. "P<0.05, compared with untreated cells. PARP, poly (ADP-ribose) polymerase; The, theanine; NS, not significant.

performed MultiCaspase apoptosis detection for evaluating theanine-induced HepG2 cell death. It was found that theanine induced the activation of caspases when the HepG 2 cells were cultured in the glutamine restricted and deprived media (Fig. 2B). During apoptosis, typical molecular events occur, including the activation of initiators and effectors of caspases, and cleavage of PARP (18). The present study examined the protein levels of PARP, procaspase- 8 and procaspase- 3 using western blot analysis (Fig. 2C). The data demonstrated that the cleavage of PARP and procaspase- 3 was increased in the theanine-treated HepG2 cells exposed to acute glutamine deprivation, whereas procaspase- 8 remained unchanged. Taken together, these results indicated that, under acute glutamine deprivation, theanine induced typical apoptotic cell death in the HepG2 cells.

Theanine treatment activates caspase-9 and caspase-3. The results described above showed that caspase cascades were involved in theanine-induced apoptosis. Two pathways have been considered to lead to caspase activation during apoptosis, 

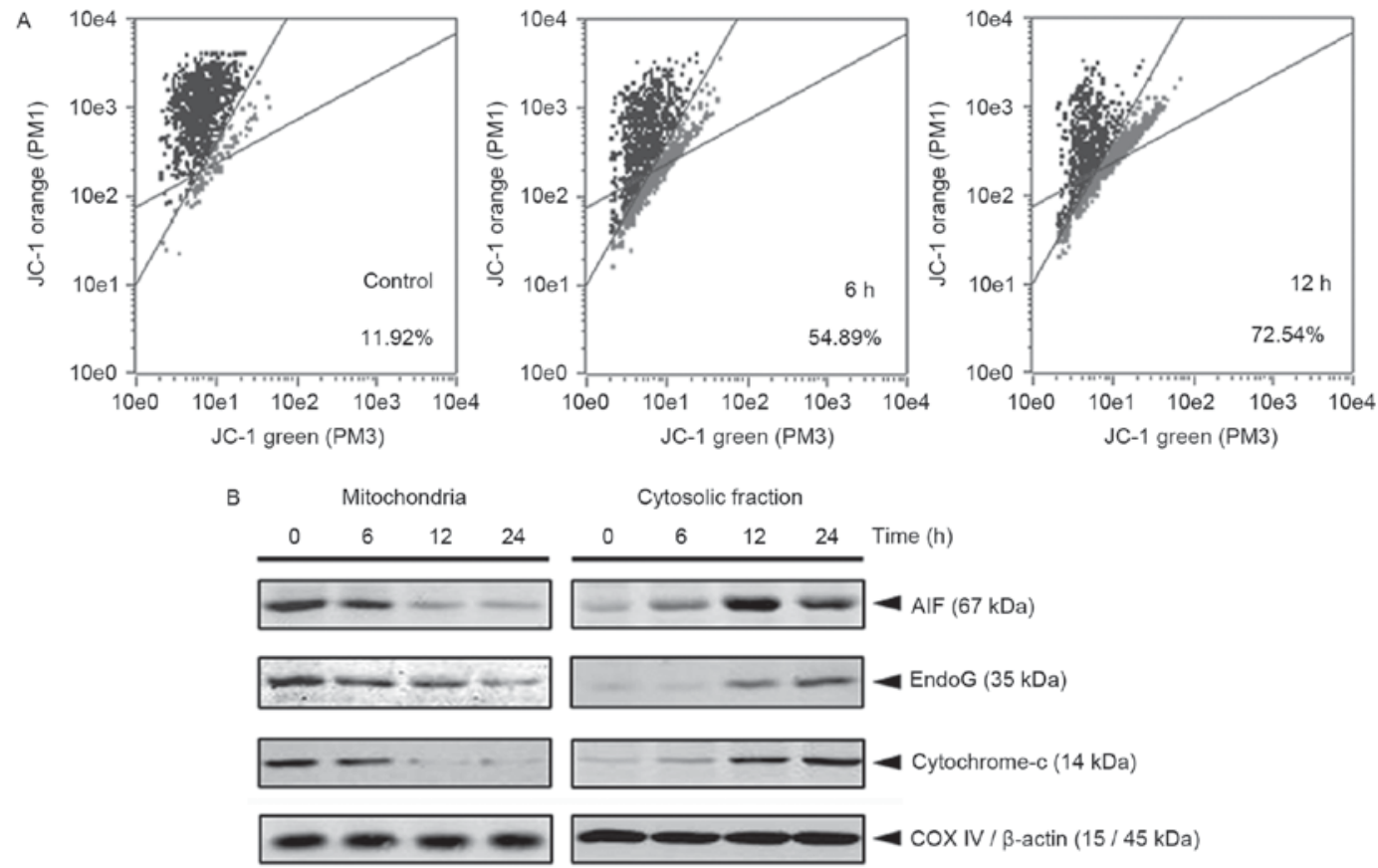

Figure 4. Activation of mitochondrial pathways in theanine-induced apoptosis. (A) HepG2 cells in glutamine-free media were treated with $300 \mu \mathrm{g} / \mathrm{ml}$ theanine for the indicated time, following which cells were prepared for measurement of MMP changes. Data of depolarized ratios are representative of three independent experiments. (B) HepG2 cells in glutamine-free media were treated with $300 \mu \mathrm{g} / \mathrm{ml}$ theanine for the indicated time. Cytoplasmic and mitochondrial proteins were separated using a mitochondria isolation kit, and the release of AIF, EndoG and cytochrome $c$ were measured using western blot analysis Levels of COX IV and $\beta$-actin were determined to confirm equal loading of mitochondrial or cytoplasmic proteins. Data shown are representative of three independent experiments. AIF, apoptosis-inducing factor; EndoG, endonuclease G; COX IV, cyclooxygenase IV.

caspase-8 and caspase-9, being major extrinsic and intrinsic initiators of caspase cascades, respectively. As shown in Fig. 2C, theanine stimulated the cleavage of caspase-3, but did not activate caspase- 8 . Therefore, the present study determined changes in the protein levels of procaspase-8, procaspase- 9 and procaspase-3 in the HepG 2 cells following theanine treatment under glutamine deprivation conditions. The results from the western blot analyses indicated that procaspase- 9 and procaspase- 3 were cleaved following theanine treatment, whereas caspase- 8 was intact (Fig. 3A). The activity of caspase- 8 was then compared with caspase- 9 using a luminescence-based assay. The data showed that, following $12 \mathrm{~h}$ incubation with theanine, the HepG2 cells exhibited 2.5 times higher caspase- 9 activity, but no change in caspase- 8 activity (Fig. 3B). Caspase inhibitors were also used to investigate the role of caspase- 9 and caspase- 8 in theanine-induced apoptosis. As shown in Fig. 3C, the caspase-8 inhibitor Z-IETD-FMK failed to prevent theanine-induced apoptosis, whereas the caspase-9 inhibitor Z-LEHD-FMK and pan caspase inhibitor Z-VAD-FMK attenuated HepG2 cell death. Further experiments showed that Z-LEHD-FMK and Z-VAD-FMK inhibited theanine-induced PARP cleavage and caspase-3 activation, whereas Z-IETD-FMK exerted minimal effect on the activation of PARP and caspase-3 (Fig. 3D). Taken together, the data demonstrated that caspase-9, but not caspase-8, was essential in the theanine-induced apoptosis. It was also noted that the pan caspase inhibitor Z-VAD-FMK did not completely prevent theanine-induced apoptosis, suggesting that caspase-independent cell death existed in this process.

Involvement of the mitochondrial pathway in theanine-induced apoptosis. In the intrinsic pathway of apoptosis, MMP is the critical event responsible for caspase activation (19). The cationic dye, JC-1, was used to evaluate alterations in MMP during theanine-induced HepG2 cell apoptosis. The results revealed $\sim 73 \%$ depolarized MMP was detected in the cells treated with $300 \mu \mathrm{g} / \mathrm{ml}$ theanine for $12 \mathrm{~h}$ (Fig. 4A). Caspase-9 activation has been reported to be associated with the mitochondrial pathway in apoptosis. Once cytochrome $c$ is released from mitochondria, it binds to cytosolic protein adapter molecule apoptosis protease activating factor 1 , which recruits and cleaves the inactive procaspase- 9 thereby promoting it into the active form. As the above data indicated that caspase- 9 was important in theanine-induced apoptosis, the present study detected the release of cytochrome $c$ from mitochondria of the HepG2 cells following theanine treatment. As expected, a time-dependent increase in cytosolic cytochrome $c$ induced by theanine was detected (Fig. 4B). It has been recognized that the release of the caspase-independent death effectors AIF and EndoG from the mitochondria is a hallmark of caspase-independent cell death. The results of the present study further demonstrated that cytosolic AIF and EndoG were increased in the theanine-treated HepG2 cells (Fig. 4B). Taken together, these data suggested that caspase- 9 and the caspase-independent mitochondrial pathway were involved in theanine-induced apoptosis.

\section{Discussion}

Liver cancer has long been considered a therapeutic challenge due to its cytotoxic drug resistant nature and associated liver function disorder, which reduces the safety of a number of conventional chemotherapeutic agents (20). In addition to 


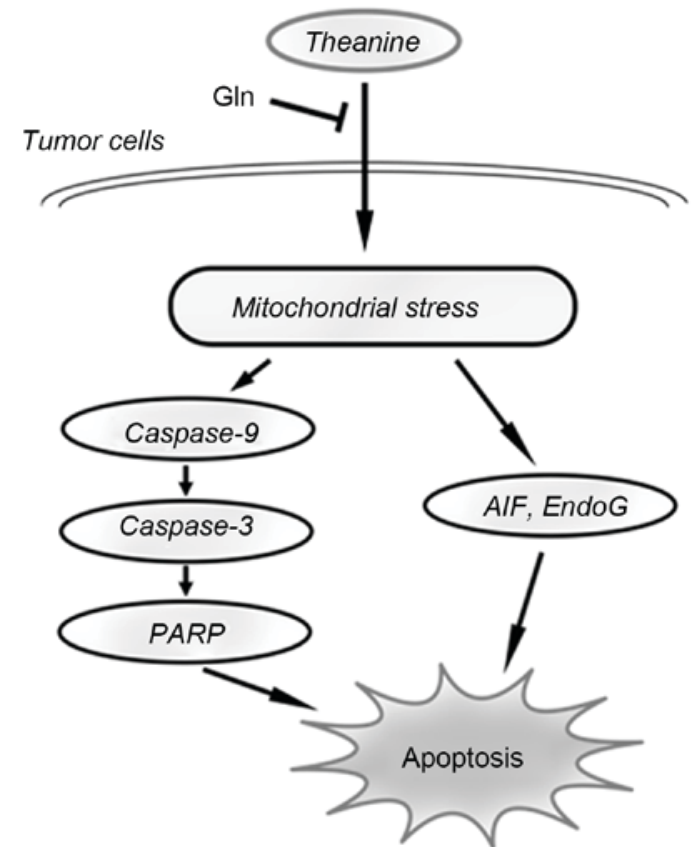

Figure 5. Schematic diagram of proposed theanine function on the tumor cell apoptotic pathway. Gln, glutamine; AIF, apoptosis-inducing factor; EndoG, endonuclease G; PARP, poly (ADP-ribose) polymerase.

cytotoxic drugs, there are certain chemicals from traditional Chinese medicine or dietary foods, which target apoptosis and ate being investigated for cancer treatment (9). In the present study, it was demonstrated that theanine, a free amino acid from green tea, induced human hepatocellular carcinoma HepG2 cell death, and this effect was significantly enhanced by acute glutamine restriction or deprivation. It was subsequently found that theanine induced HepG2 cell death through apoptosis.

In the present study, it was found that a concentration $>300 \mu \mathrm{g} / \mathrm{ml}$ of theanine was required for inducing significant cell apoptosis when the cells were cultured in DMEM supplemented with $4 \mathrm{mM}$ glutamine (Fig. 1A). It is reasonable to suggest that glutamine antagonized the apoptotic effect of theanine due to their similar structures. The present study then imposed acute restriction/deprivation of glutamine, and marked enhancement in the efficacy of theanine in apoptosis was induced. This finding is consistent with those of a previous report, which demonstrated that the accumulation of theanine in the brain synaptosomal fraction was markedly inhibited by glutamine, whereas glutamine accumulation was inhibited by theanine and vice versa (16). Therefore, it was hypothesized that glutamine interfered with the action of theanine in tumor cells. In addition, the results also showed that the acute restriction/deprivation of glutamine alone within $24 \mathrm{~h}$ did not lead to nutrient withdrawal stress towards the HepG2 and HeLa cells (Fig. 1B and C).

In various clinical situations, appropriate exogenous glutamine supply is considered to contribute beneficially to reduce risks of high-dose chemotherapy (21). Similarly, theanine has been shown to protect normal cells from damage by chemotherapeutic drugs (22). As theanine is a glutamate derivative, it may be enzymatically metabolized to glutamate in the liver, and consequently be utilized as a constituent of glutathione (GSH). Therefore, theanine can attenuate the DOX-induced adverse reactions involved in oxidative damage, due to increase in glutamate and the recovery of GSH levels in normal tissues $(15,22)$. However, tumor cells rapidly consume glutamine and use it to supply metabolic pathways, which support cell growth and proliferation. Glutamine is also a multipurpose nutrient, feeding several additional pathways, which enhance the ability of cells to communicate with each other, and to cope with stress by oncogenic signaling and the tumor microenvironment (23). Therefore, oncologists often avoid supplying glutamine to tumor-bearing host to avoid any potential risk.

In the present study, the cytotoxicity of theanine towards five cell lines, including two tumor cell lines and three types normal cell lines, was examined. Consistent with the above, the apparent cytotoxicity of theanine towards HepG2 and HeLa cells was detected when glutamine was restricted in the medium, and the higher cytotoxicity of theanine was exhibited in HepG2 cells. Therefore, theanine may be a valuable therapeutic reagent when supplementation of glutamine is refused to avoid interference during treatment.

The understanding of apoptosis has provided the basis for novel targeted therapies, which can induce cancer cell death or sensitize cells to established cytotoxic agents and radiation therapy. In the present study, it was found that caspase-9, but not caspase-8, was involved in theanine-induced HepG2 cell apoptosis. As is commonly known, apoptosis occurs through two main pathways, which includes the extrinsic (cytoplasmic) pathway triggered through death receptor and intrinsic (mitochondrial) pathway, which leads to the release of cytochrome $c$ from the mitochondria and activation of the death signal. Caspase- 8 and caspase- 9 are important in the extrinsic and intrinsic apoptotic pathways, respectively (18). To clarify the underlying mechanism of theanine-induced apoptosis, the present study measured the activation of caspase-3, MMP, release of cytochrome $c$, and protein levels of AIF and EndoG. These experiments demonstrated that theanine induced HepG2 cell apoptosis by activating the intrinsic apoptotic pathway. Sadzuka et al (22) demonstrated that glutaminase reacted with theanine to generate glutamate in vitro, and that the metabolism of theanine mediated by glutaminase, and the increase of glutamate-mediated GSH is important for theanine anticancer action in vivo. HepG2 cells accumulate higher levels of glutamine, compared with normal cells, and are more sensitive to glutamine withdrawal from the medium (24). When extrinsic glutamine is restricted, it may be easier for theanine to disturb glutamine/glutamate metabolism and trigger the generation of inappropriate metabolites, followed by activation of the intrinsic apoptosis pathway. In mammals, glutaminase exists in the matrix of mitochondria, therefore, it was expected that theanine may alter glutamine/glutamate metabolism at the site of mitochondria. Taken together, the results of the present study indicated the function of theanine on cancer cells (Fig. 5). Further investigations are required to demonstrate the intracellular interference between theanine and glutamine, and the signal transduction networks underlying the theanine-induced apoptosis.

In conclusion, the present study showed that theanine induced HepG2 cell death under acute glutamine restriction/deprivation 
conditions by triggering caspase-9-dependent and caspase-independent mitochondrial pathways. It was found that glutamine restriction and/or deprivation amplified the apoptotic action of theanine in HepG2 cells. These observations shed light on the potential use of theanine in the therapeutic treatment of liver cancer and support the view that glutamine supplementation requires cautious use during chemotherapy.

\section{Acknowledgements}

Not applicable.

\section{Funding}

The present study was supported by the National Nature Science Foundation of China (grant no. 81471557), the Jiangsu Perspective Study (grant no. BY2013001-03), the Natural Science Research Project of Anhui Provincial Education Department (grant no. KJ2017A908) and the Science Research Project of Chuzhou City Vocation College (grant no. 2016zk02).

\section{Availability of data and materials}

All data generated or analyzed during this study are included in this published article.

\section{Authors' contributions}

YX was responsible for the experimental design and implementation. PB was a major contributor in writing the manuscript and conducted some of the experiments. QW was responsible for image processing and data analysis. YZ was responsible for cell culture and performed the experiments. ZY and LL interpreted the data. All authors read and approved the final manuscript.

\section{Ethics approval and consent to participate}

Not applicable.

\section{Patient consent for publication}

Not applicable.

\section{Competing interests}

The authors declare that they have no competing interests.

\section{References}

1. Liang YR, Liu C, Xiang LP and Zheng XQ: Health benefits of theanine in green tea: A review. Trop J Pharmaceut Res 14: $1943-1949,2015$

2. Terashima T, Takido J and Yokogoshi H: Time-dependent changes of amino acids in the serum, liver, brain and urine of rats administered with theanine. Biosci Biotechnol Biochem 63: 615-618, 1999.
3. Juneja LR, Chu DC, Okubo T, Nagato Y and Yokogoshi H: L-theanine-a unique amino acid of green tea and its relaxation effect in humans. Trends Food Sci Technol 10: 199-204, 1999.

4. Gomez-Ramirez M, Kelly SP, Montesi JL and Foxe JJ: The effects of L-theanine on alpha-band oscillatory brain activity during a visuo-spatial attention task. Brain Topogr 22: 44-51, 2009.

5. Cho HS, Kim S, Lee SY, Park JA, Kim SJ and Chun HS: Protective effect of the green tea component, L-theanine on environmental toxins-induced neuronal cell death. Neurotoxicology 29: 656-662, 2008.

6. Bozzetti F, Biganzoli L, Gavazzi C, Cappuzzo F, Carnaghi C, Buzzoni R, Dibartolomeo $\mathrm{M}$ and Baietta E: Glutamine supplementation in cancer patients receiving chemotherapy: A double-blind randomized study. Nutrition 13: 748-751, 1997.

7. Yokogoshi $\mathrm{H}$ and Kobayashi M: Hypotensive effect of gamma-glutamylmethylamide in spontaneously hypertensive rats. Life Sci 62: 1065-1068, 1998.

8. Zhang G, Ye X, Ji D, Zhang H, Sun F, Shang C, Zhang Y, Wu E, Wang F, Wu F, et al: Inhibition of lung tumor growth by targeting EGFR/VEGFR-Akt/NF- $\kappa$ B pathways with novel theanine derivatives. Oncotarget 5: 8528-8543, 2014.

9. Martin KR: Targeting apoptosis with dietary bioactive agents Exp Biol Med (Maywood) 231: 117-129, 2006.

10. Nakazato T, Ito K, Miyakawa Y, Kinjo K, Yamada T, Hozumi N, Ikeda Y and Kizaki M: Catechin, a green tea component, rapidly induces apoptosis of myeloid leukemic cells via modulation of reactive oxygen species production in vitro and inhibits tumor growth in vivo. Haematologica 90: 317-325, 2005.

11. Yang GY, Liao J, Kim K, Yurkow EJ and Yang CS: Inhibition of growth and induction of apoptosis in human cancer cell lines by tea polyphenols. Carcinogenesis 19: 611-616, 1998.

12. Kundu T, Dey S, Roy M, Siddiqi M and Bhattacharya RK: Induction of apoptosis in human leukemia cells by black tea and its polyphenol theaflavin. Cancer Lett 230: 111-121, 2005.

13. Zhang G, Miura Y and Yagasaki K: Inhibitory effects of theanine and sera from theanine-fed rats on receptor-mediated cancer cell invasion beneath mesothelial-cell monolayers. Cytotechnology 36: 195-200, 2001.

14. Liu Q, Duan H, Luan J, Yagasaki K and Zhang G: Effects of theanine on growth of human lung cancer and leukemia cells as well as migration and invasion of human lung cancer cells. Cytotechnology 59: 211-217, 2009.

15. Sugiyama $\mathrm{T}$ and Sadzuka $\mathrm{Y}$ : Theanine, a specific glutamate derivative in green tea, reduces the adverse reactions of doxorubicin by changing the glutathione level. Cancer Lett 212: 177-184, 2004.

16. Kakuda T, Hinoi E, Abe A, Nozawa A, Ogura M and Yoneda Y: Theanine, an ingredient of green tea, inhibits $[3 \mathrm{H}]$ glutamine transport in neurons and astroglia in rat brain. J Neurosci Res 86: 1846-1856, 2008

17. Friedman M, Mackey BE, Kim HJ, Lee IS, Lee KR, Lee SU, Kozukue E and Kozukue N: Structure-activity relationships of tea compounds against human cancer cells. J Agric Food Chem 55: 243-253, 2007.

18. Shi Y: Mechanisms of caspase activation and inhibition during apoptosis. Mol Cell 9: 459-470, 2002.

19. Kroemer G, Galluzzi L and Brenner C: Mitochondrial membrane permeabilization in cell death. Physiol Rev 87: 99-163, 2007.

20. Kerr SH and Kerr DJ: Novel treatments for hepatocellular cancer. Cancer Lett 286: 114-120, 2009.

21. Kuhn KS, Muscaritoli M, Wischmeyer P and Stehle P: Glutamine as indispensable nutrient in oncology: Experimental and clinical evidence. Eur J Nutr 49: 197-210, 2010.

22. Sadzuka Y, Sugiyama T, Nagamine M, Umegaki K and Sonobe T: Efficacy of theanine is connected with theanine metabolism by any enzyme, not only drug metabolizing enzymes. Food Chem Toxicol 44: 286-292, 2006.

23. Shanware NP, Mullen AR, DeBerardinis RJ and Abraham RT: Glutamine: Pleiotropic roles in tumor growth and stress resistance. J Mol Med (Berl) 89: 229-236, 2011.

24. Tardito S, Chiu M, Uggeri J, Zerbini A, Da Ros F, Dall'Asta V, Missale $\mathrm{G}$ and Bussolati O: L-Asparaginase and inhibitors of glutamine synthetase disclose glutamine addiction of $\beta$-catenin-mutated human hepatocellular carcinoma cells. Curr Cancer Drug Targets 11: 929-943, 2011. 